\title{
THE EXPANDING ROLE OF IMMERSIVE MEDIA IN EDUCATION
}

\author{
Emre Erturk and Gabrielle-Bakker Reynolds \\ Eastern Institute of Technology, 501 Gloucester Street, Taradale, Napier 4112, New Zealand
}

\begin{abstract}
This paper seeks to understand the impact of immersive media, in particular augmented reality, within the education sector. A small experimental methodology was adopted alongside a current literature review. Three research questions were posited as follows: (1) What are the affordances of immersive media within education? (2) What are the barriers to immersive media in education? (3) How may immersive media be implemented?

Affordances of immersive media include greater content customisation, increased creativity with less risk, promoting student interaction, enhanced motivation and engagement, and the chance for students to engage in extraordinary experiences. In 2020, many students around the world are staying home and studying in isolation, as a result of the COVID-19 pandemic. In this emergency context, mobile applications can offer interesting experiences that can make life and learning less difficult and more pleasant.

The mobile web application Metaverse was used to exemplify in two different ways how immersive media can be appropriately used as a tool in a university level education setting. The first prototype was a short quiz on general knowledge about New Zealand, to help orient new international students. The second prototype was a quick poll to feel the pulse and understand the mood of students while at home and physically away from the campus and their lecturers. In summary, the authors present an updated review of this technology and present their own new examples of applications that serve additional purposes.
\end{abstract}

\section{KEYWORDS}

Immersive Media, Augmented Reality, Mobile Learning, Home Learning, Educational Technology

\section{INTRODUCTION}

Immersive media can be understood as any technology that blends computer generated content with the physical environment, creating a sense of immersion and stimulating the human senses through interactive multimedia. For instance, virtual reality aims to occlude the physical environment. In contrast, augmented reality may involve overlaying content the upon physical world. The Virtuality Continuum (see Figure 1) was created in 1994 by Milgram and Kishino as cited in Milman (2018) to show the various modes on a scale.

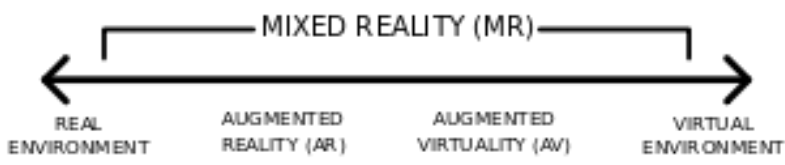

Figure 1. The Virtuality Continuum

Since immersive media's use within the educational sector is still relatively recent, the objective of this paper is to explore its role by discussing its pedagogical affordances and barriers, and provide examples of its practical use. Common previous applications of augmented reality were for younger students and children, or for teaching high school and university students application development and visual design. This paper takes a broader approach in a different context in 2020 where virtual engagement is important. 


\section{LITERATURE REVIEW}

The literature review examined peer-reviewed sources. Keywords used to find sources include "immersive media," "immersive technologies," "immersive media and education," "augmented reality and education" "virtual reality and student learning," "mixed reality and education" and "AR, VR, MR, and education."

Jensen \& Konradsen (2018) undertook research about the use of virtual reality head-mounted displays in education and training and their findings demonstrated several instances where virtual reality assisted in skills acquisition related to cognitive abilities. Furthermore, Sirakaya (2018) explored the effects of augmented reality use on achievement, misconception, and course engagement among 118 seventh grade students within six different classes. They concluded that students' levels of engagement were not necessarily affected by the implementation of augmented reality. Nevertheless, overall, the students' achievement levels were increased by augmented reality, while reducing any misconceptions from theoretical learning.

Harper et al. (2018) developed a mixed reality game made for simulation-based training, specifically, stonemasonry. Currently, vocational training can be restricted by regulations that do not permit the use of power tools, such as an angle grinder. So, the use of cyber-physical systems allows students to learn without the safety risks. A prototype of a mixed reality, vocational educational training (VET) game was created using Microsoft HoloLens, receiving positive feedback during the trials.

Team-based teaching is another possible approach. Denholm et al. (2013) conducted a study on the value of team-based mixed reality games in higher education on international students. Results demonstrated that not only did $75 \%$ of students believe that the mixed reality games improved team collaboration, but over $60 \%$ indicated that participation in mixed-reality games were more useful than lectures.

Furthermore, Cascales et al. (2013) examined parents' perceptions of the use of didactical augmented reality in preschool education. They categorised parents' perceptions into elements: writing, reading, knowledge, motivation, degree of satisfaction, and creativity. Although findings showed differences among the participants, the majority of parents believed that augmented reality offered benefits.

\section{METHODOLOGY}

The first part of Section 4 covers two questions based on a literature review. To address these questions, the authors examined a range of case studies, industry-based information, and educator perspectives. The method of search utilised different resources and online databases such as Google Scholar, ProQuest, and ERIC. Section 4.2 summarizes the experimental project for the third question by investigating the use of the augmented reality platform Metaverse Studio, which is an open-source web platform that allows users to create interactive experiences without the need for coding. Two applications have been created to show how Metaverse may be used as an educational tool. The research questions for the next section are: (1) What are the affordances of immersive media within education? (2) What are the barriers to immersive media within education? and (3) How may immersive media be implemented in the education sector?

\section{FINDINGS}

\subsection{Affordances of Immersive Media and the Barriers}

Findings suggest that the pedagogical benefits of immersive media in education include greater customisation, increased creativity and less risk, promotion of student interaction, enhanced motivation and engagement, and extending traditional learning by offering experiences that would otherwise be impossible.

Fernandez (2017) investigated how virtual reality can be used as a tool in representing abstract concepts. Virtual reality is able to be programmed so that situations can be controlled, as well as contexts in which students act. Applications can be tailored to suit a diverse range of subjects and geographical locations. Augmented reality increases creativity among students because it offers fewer risks. Within teaching health, an image of a human body may be superimposed in a classroom environment and split into different segments. In engineering, a digital model of an engine may be superimposed and examined in different ways. 
Khan et al. (2019) researched the impact of augmented reality on the learning motivations of health science students at the University of Cape Town. Results supported the notion that augmented reality assists in terms of motivation, and helps bring an increase in students' attention, confidence, and satisfaction.

Google Cardboard, a virtual reality platform, can be used for learning (https://arvr.google.com/cardboard/). For instance, the Expeditions application offers tours around the world, allowing teachers to take students on virtual field trips (https://edu.google.com/products/vr-ar/expeditions/). This enables students to undergo experiences that would otherwise be dangerous, impossible, or costly, such as swimming with sharks or visiting space. It allows a level of customisation that would not be possible with physical tours, and in turn can be more engaging for student learning. Reinforcing this, Foote (2017) found that immersive technologies such as Google Cardboard are valuable tools for teaching geography because they may increase engagement and heighten students' motivation to learn. Lastly, Knierim et al. (2018) explored mixed reality in education, highlighting (1) the personalisation of learning, (2) strengthening of ubiquitous learning through spatially distributed knowledge, and (3) enrichment of learning by amplifying students' understanding of course materials. These findings may be more representative of how students react to a new technology, and less representative of whether the benefits may continue in the long term.

Overall, a final significant affordance of immersive media may be how it enables learning experiences that would otherwise be costly. This may be considered as an incentive for its adoption in education.

Barriers to immersive media include lack of teacher competencies and pedagogical structure in some applications, cognitive overload among students, inflexibility of immersive applications, and technical issues.

Martins et al. (2015) investigated the challenges of using augmented reality within music education, noting that barriers towards augmented reality's adoption include technological issues. For instance, designing applications is not a competence that all teachers have. Augmented reality applications in the educational sector are often influenced by game developers and may not always be pedagogically structured.

Furthermore, Wu et al. (2013) examined the challenges that come with employing augmented reality within education, noting that learning issues may be a barrier. For instance, students have reported feeling overwhelmed. Some tasks in augmented reality applications may be challenging if instructions are missing. This points to another issue teachers may face: the inflexible design of some augmented reality applications.

In relation to the challenges, Akçayır \& Akçayır (2017) conducted a systematic literature review, noting technical issues as a hindrance, e.g. location-based applications using GPS may miscalculate directions or locations. Some applications offer low sensitivity in trigger detection. Similar issues are pointed out in Knierim et al.'s (2018) research into the use of mixed-reality, e.g. lack of comfortability with prolonged wear and limited field of view. Hands-free interaction is scarcely supported; hand gestures and speech are often required, and mid-air hand gestures may cause fatigue. Overall, because the technology is constantly improving, what is learned from past studies is not conclusive and new research is expected on this topic.

\subsection{Immersive Media Implementation}

In this section, the authors demonstrate recent uses of augmented reality in a university level education setting. Both sample applications were created using the web platform Metaverse (https://studio.gometa.io/). The requirement for users is that they need to have the Metaverse app on their mobile device to scan a $Q R$ code to play the experiences, which overlays the multimedia elements over the physical environment.

The idea behind the first experience was an enjoyable quiz on general knowledge and trivia about New Zealand, especially for new international students. The questions are not easy; therefore, this quiz can be played by the general public as well. A friendly cartoon face is overlaid on the mobile device screen and asks the questions one by one - allowing students to guess again before moving on to the next question if they give an incorrect answer. Students can also end the experience if they wish. The quiz adds the points for each user, and generates a leaderboard (scoreboard). This provides an incentive to play again.

According to the feedback, there have been two advantages to this augmented reality approach (as opposed to having a web browser-based quiz). First, the students can run this without requiring a computer, and in any comfortable setting with their phones, for example, outdoors. Secondly, this experience can be enjoyed together with others. In the case of a mature student, this can be their family (e.g. spouse and child).

The second experience is a poll taken during the 2020 global pandemic and the ensuing lockdown, when students have found themselves in a situation that is also different from ordinary online study. For example, most students studying online would still have access to campus or education centre resources and 
face-to-face advisors, which are now lacking. Therefore, the authors released an augmented reality app to take the pulse of postgraduate IT students. In the app, the camera turns on and an interactive friendly robot is overlaid and floats around in the room. It conducts a quick poll, and gives each student advice based on their mood.

Both applications have been tested with users, successfully without technical issues. The tests can be discussed in a longer paper in the future. The results of the poll are anonymous, and have shown most students to be happy or normal in their study bubble. The experience offers them the chance to call someone if they are feeling unhappy or having issues; although students have not selected this option. Another lesson learned from both apps is that creating an augmented reality experience is not difficult in Metaverse. This is not only an educators' tool, but also a tool that students can use as a general creative outlet away from school.

\section{CONCLUSION}

Immersive media has benefits such as: enhanced motivation, and extending traditional teaching practices by engaging students in different ways. The literature and numerous trials support the idea that immersive media offers new opportunities to interact. Barriers such as lack of teacher competencies and lack of pedagogical structure and inflexibility of applications may be a result of a divide between educators and developers. Therefore, it is recommended to have more communication between teachers and developers. Lack of certain competencies can be addressed by technical training for teachers. This project has also trialed different practical uses of immersive media and illustrated its value. Although the work continues to progress, the authors have prepared to share screenshots of their current applications during the conference. If new applications are created, they will boost educators' experience and increase the implementation in this realm.

\section{REFERENCES}

Akçayır, M. and Akçayır, G. (2017). Advantages and Challenges Associated with Augmented Reality for Education: A Systematic Review of the Literature. Educational Research Review, Vol. 20, pp. 1-11. https://doi.org/10.1016/j.edurev.2016.11.002

Cascales et al. (2013). Study on Parents' Acceptance of the Augmented Reality Use for Preschool Education. Procedia Computer Science, Vol. 25, pp. 420-427. https://doi.org/10.1016/j.procs.2013.11.053

Denholm et al. (2013). The Value of Team-based Mixed-reality (TBMR) Games in Higher Education. International Journal of Game Based Learning, Vol. 3, No 1, pp. 18-33.

Fernandez, M. (2017). Augmented Virtual Reality: How to Improve Education Systems. Higher Learning Research Communications, Vol. 7, No. 1, pp. 1-15. https://doi.org/10.18870/hlrc.v7i1.373

Foote, C. (2017). Mobile technology goes virtual: using virtual reality in education. Internet at Schools, Vol. 24, No. 3.

Harper et al. (2018). Development of a Mixed Reality Game for Simulation Based Education. Proceedings of 12th European Conference on Games Based Learning. Sophia Antipolis, France, pp. 212-220.

Jensen, L. and Konradsen, F. (2018). A Review of the Use of Virtual Reality Head-mounted Displays in Education and Training. Educational Technology, Research and Development, Vol. 66, No. 5, pp. 1515-1529.

Khan et al. (2019). The Impact of an Augmented Reality Application on Learning Motivation of Students. Advances in Human-Computer Interaction. https://doi.org/10.1155/2019/7208494

Knierim et al. (2018). Challenges and Opportunities of Mixed Reality Systems in Education. https://dx.doi.org/10.18420/muc2018-ws07-0471

Martins et al. (2015). Challenges and Possibilities of Use of Augmented Reality in Education Case Study in Music Education. Proceedings of 15th International Conference on Computational Science and Applications. Banff, Canada, pp. 223-233.

Milman, N. (2018). Defining and Conceptualising mixed reality, Augmented Reality, and Virtual Reality. Distance Learning, Vol. 15, No. 2, pp. 5-58.

Sirakaya, M. (2018). The Effect of Augmented Reality Use on Achievement, Misconception and Course Engagement. Contemporary Educational Technology, Vol. 9, No. 3, pp. 297-314.

$\mathrm{Wu}$ et al. (2013). Current Status, Opportunities and Challenges of Augmented Reality in Education. Computers and Education, Vol. 62, pp. 41-49. https://doi.org/10.1016/j.compedu.2012.10.024 